\title{
Subclinical systemic lymphedema in patients with obesity and lipedema
}

\begin{abstract}
Obesity is a clinical condition that affects millions of people around the world and is associated with inflammatory processes. The aim of the present study was to report the association between obesity, lipedema, and systemic fluid retention, characterizing subclinical systemic lymphedema with aggravating factors. A 50-year-old female patient weighing 150 kilograms (body mass index: $60.2 \mathrm{~kg} / \mathrm{m}^{2}$ ) reported being obese since childhood, but more located on the hips. She had a family history of this body configuration. Electrical bioimpedance analysis revealed generalized edema, constituting systemic lymphedema. Subclinical systemic lymphedema is caused by obesity and lipedema is also associated with this condition.
\end{abstract}

\section{Keywords: lymphedema, lipedema, obesity, inflammation}

\section{Introduction}

Obesity is a clinical condition that affects millions of people around the world and is associated with inflammatory processes [1]. Clinical weight loss is the best option, but surgical procedures, such as bariatric surgery, have emerged over the years. Lymphedema is a clinical condition involving dysfunction of the lymphatic system affecting both the formation and drainage of lymph. The accumulation of macromolecules in the interstitial space is associated with the retention of fluids and the consequent formation of edema. Treatment involves therapies that mobilize these macromolecules, such as specific lymphatic therapeutic techniques and compression mechanisms combined with exercise and lymphomyokinetic activities [2].

Lipedema is a physical aspect characterized by greater fat distribution in the upper and lower limbs. It is not characterized as a disease and occurs in approximately $10 \%$ of the population. As the main physiopathological change, however, such individuals can develop lymphostasis and fat necrosis [3].

Animal studies have demonstrated that the progression of obesity is associated with changes in the lymphatic system involving the pumping mechanism (lymphangions), an inflammatory process as well as changes in capillary permeability and immunological defense $[4,5]$. A case study involving lymphoscintigraphy reports an association between lower limb lymphedema and both lymphatic changes and clinical lymphedema [6]. Subclinical systemic lymphedema caused by obesity manifests earlier in patients with lymphedema and worsens with the progression of obesity [7].

Electrical bioimpedance analysis has revealed a greater amount of intracellular and extracellular water in cases of lymphedema that can involve segments of the body or the entire body itself. The aim of the present study was to report a case of obesity, lipedema, and lymphatic dysfunction.

\section{Case Report}

A 50-year-old female patient weighing 150 kilos and with a body mass index of $60.2 \mathrm{~kg} / \mathrm{m}^{2}$ reported being obese since childhood, but more located on the hips. She reported losing weight in adolescence but became obese again soon afterward. She had a family history of the body configuration. The patient sought our clinic due to leg pain. The physical examination revealed obesity with lipedema located in the thighs and hips, edema in the legs and no important edema in the feet. Electrical bioimpedance analysis revealed generalized edema, characterizing
Jose Maria Pereira de Godoy ${ }^{1 *}$, Henrique Jose Pereira de Godoy², Ana Carolina Pereira de Godoy ${ }^{3}$ and Maria de Fatima Guerreiro Godoy ${ }^{4}$

${ }^{1}$ Department of Cardiology and Cardiovascular Surgery, Medicine School, São José do Rio Preto (FAMERP), National Council for Research and Development, Brazil

${ }^{2}$ Medicine School of Universidade Federal do Mato Grosso-Cuiabá-UFMT and Research Group of Clínica Godoy, Sao Jose do Rio Preto, Brazil

${ }^{3}$ Pediatrics Unit Intensive Therapy of Santa Casa de São Paulo, Brazil and Research Group of Clínica Godoy, São Jose do Rio Preto, Brazil

${ }^{4}$ Medicine School, São José do Rio Preto (FAMERP), Clínica Godoy, Brazil

*Author for correspondence:

godoyjmp@gmail.com 
TABLE 1. Intracellular and extracellular fluid, fluid in limbs and trunk and reference values.

\begin{tabular}{|c|c|c|c|}
\hline & Total & Normal water values & Total extracellular water/total body water ratio \\
\hline Total intracellular water & 28.1 & 16.9 to 20.7 & \\
\hline Total extracellular water & 19.5 & 10.4 to 12.6 & \\
\hline Total extracellular water/total body water ratio & 0.41 & 0.36 to 0.39 & 0.384 limit $(0.36-0.39)$ \\
\hline Right arm & 2.46 & 1.39 to 1.69 & 0.388 limit $(0.36-0.39)$ \\
\hline Left arm & 2.41 & 1.39 to 1.695 & 0.399 limit $(0.36-0.39)$ \\
\hline Trunk & 20.0 & 12.6 to 15.4 & 0.399 limit $(0.36-0.39)$ \\
\hline Right leg & 7.28 & 4.37 to 5.35 & 0.419 limit $(0.36-0.39)$ \\
\hline
\end{tabular}

systemic lymphedema with more intracellular and extracellular water in the limbs and thorax. It is noteworthy that the bioimpedance evaluation revealed lower limb lymphedema without the presence of edema below the knee TABLE 1, characterizing subclinical systemic lymphedema. This is an intermediate stage involving the increase in bodily fluids without the progression to lymphedema of the upper limbs but in the trunk and lower limbs.

\section{Discussion}

The present study describes a patient with lipedema and morbid obesity as well as generalized fluid retention that we denominate subclinical systemic lymphedema, which is a novel concept of lymphedema. The lymphatic system is a functional reserve of the venous system and edema emerges when this reserve is surpassed. In such cases, edema is generalized in all limbs and the thorax.

Animal studies have demonstrated an association between the progression of obesity and changes in the lymphatic system involving the pumping mechanism (lymphangions), capillary permeability and changes in the immune response [3-5]. These studies report findings that suggest mechanical changes involving a reduction in the pumping of the lymphatic system, which is a dynamic process characterized by changes in capillary permeability. A case study involving lymphoscintilography found an association between obesity and lymphatic dysfunction that were not reversed with weight loss, suggesting that obesity may cause irreversible harm to the lymphatic system.

Regarding lipedema, studies have detected lymphostasis, which may have contributed to the systemic edema found in the patient described herein. No lymphedema was found below the knee and the edema was concentrated in the thigh. Therefore, the diagnosis was thigh lymphedema caused by obesity and the lymphostasis of the lipedema. These findings suggest microcirculation lymphedema in patients with lipedema that may become aggravated by inflammatory processes and the involvement of the lymphatic system in cases of obesity. The interference of gravitational pressure may also contribute to the greater aggression to the lower limbs, constituting a multifactor etiology of this edema. Other conditions that affect capillary permeability should also be considered in patients with lymphedema, such as idiopathic cyclic edema [8].

With regard to treatment, weight loss and active exercise are the most indicated option. However, there is a need for studies identifying a medication option for the control of permeability and lymphatic dysfunction.

\section{Conclusion}

Subclinical systemic lymphedema is associated with obesity, but lipedema and negative interferences on venolymphatic circulation contribute to the aggravation of the edema. 


\section{REFERENCES}

Rodríguez CP, González MC, AguilarSalinas CA, et al. peripheral lymphocytes, obesity, and metabolic syndrome in young adults: An immunometabolism study. Metab Syndr Relat Disord. 29, 16 (2018).

Pereira De GJM, Amador FBP, Buzato $\mathrm{E}$, et al. Intensive outpatient treatment of elephantiasis. Int Angiol. 31, 494-498 (2012).

Halk AB, Damstra RJ. First Dutch guidelines on lipedema using the international classification of functioning, disability and health. Phlebology. 32, 152159 (2017).

Nitti MD, Hespe GE, Kataru RP, et al. Obesity-induced lymphatic dysfunction is reversible with weight loss. J Physiol. 594, 7073-7087 (2016).

García NGD, Cuzzone DA, Albano NJ, et al. Obesity but not high-fat diet impairs lymphatic function. Int $J$ Obes (Lond). 40, 1582-1590 (2016).

Greene AK, Grant FD, Slavin SA, et al. Obesity-induced lymphedema: Clinical and lymphoscintigraphic features. Plast Reconstr Surg. 135, 1715-1719 (2015).

Pereira de Godoy JM, Pereira de Godoy LM, Pereira de Godoy AC, et al. Bariatric surgery and the evaluation of subclinical systemic lymphedema. J Surg Case Rep. 2, 1-3 (2019).

Pereira de Godoy JM, Pereira de Godoy HJ, Pereira de Godoy LM, et al. Prevalence of idiopathic cyclic edema in women with lower limb lymphedema. $J$ Clin Med. 7 (2017). 